PROCEEDINGS OF THE AMERICAN MATHEMATICAL SOCIETY

Volume 124, Number 9, September 1996

\title{
ON THE HIGHER DELTA INVARIANTS OF A GORENSTEIN LOCAL RING
}

\author{
YUJI YOSHINO
}

(Communicated by Wolmer V. Vasconcelos)

To the memory of Professor Maurice Auslander

\begin{abstract}
Let $(R, \mathfrak{m})$ be a Gorenstein complete local ring. Auslander's higher delta invariants are denoted by $\delta_{R}^{n}(M)$ for each module $M$ and for each integer $n$. We propose a conjecture asking if $\delta_{R}^{n}\left(R / \mathfrak{m}^{\ell}\right)=0$ for any positive integers $n$ and $\ell$. We prove that this is true provided the associated graded ring of $R$ has depth not less than $\operatorname{dim} R-1$. Furthermore we show that there are only finitely many possibilities for a pair of positive integers $(n, \ell)$ for which $\delta_{R}^{n}\left(R / \mathfrak{m}^{\ell}\right)>0$.
\end{abstract}

\section{INTRODUCTION}

Let $(R, \mathfrak{m}, k)$ be a commutative Noetherian Gorenstein complete local ring. Maurice Auslander has introduced the delta invariant $\delta_{R}(M)$ for a finitely generated $R$-module $M$. It is defined to be the smallest integer $\mu$ such that there is an epimorphism $X \oplus R^{\mu} \longrightarrow M$ with $X$ a maximal Cohen-Macaulay module with no free summands. For an integer $n \geqq 0$, the $n$th delta invariant $\delta_{R}^{n}(M)$ is also defined by Auslander, Ding and Solberg [2] as $\delta_{R}^{n}(M)=\delta_{R}\left(\Omega_{R}^{n}(M)\right)$, where $\Omega_{R}^{n}(M)$ denotes the $n$th syzygy module of $M$ over $R$. On the other hand, S. Ding ([3], [4] and [5]) studies the delta invariants of $R / \mathfrak{m}^{\ell}(\ell \geqq 1)$ and defines an interesting, new invariant - the index of $R$.

In this paper we are particularly interested in the higher delta invariants of $R / \mathfrak{m}^{\ell}$, that is, $\delta_{R}^{n}\left(R / \mathfrak{m}^{\ell}\right)$ for $n, \ell \geqq 1$. We would like to propose the following conjecture:

Conjecture (0.1). For any positive integers $n$ and $\ell$, we would have $\delta_{R}^{n}\left(R / \mathfrak{m}^{\ell}\right)=0$ unless $R$ is regular.

Actually, as one of the main theorems of this paper, we shall show this conjecture is true if the associated graded $\operatorname{ring} g r_{\mathfrak{m}}(R)$ has depth $\geqq \operatorname{dim} R-1$. See Corollary (2.3). In particular, it is valid if $R$ is either a ring of hypersurface or a homogeneous graded ring. We note that, if $d=\operatorname{dim}(R)$, then that $\delta_{R}^{d}\left(R / \mathfrak{m}^{\ell}\right)=0$ exactly means that the $d$ th syzygy module of $R / \mathfrak{m}^{\ell}$ has no free direct summand. Hence our result generalizes a result of Herzog [6, Cor (2.4)].

Received by the editors January 20, 1995 and, in revised form, March 9, 1995.

1991 Mathematics Subject Classification. Primary 13C14, 13D02, 13H10, 16 G50.

Key words and phrases. Cohen-Macaulay modules, Gorenstein ring, Cohen-Macaulay approximations. 
Furthermore we can show in Theorem (2.1) that, in general, there are only finitely many possibilities for a pair $(n, \ell)$ of positive integers for which $\delta_{R}^{n}\left(R / \mathfrak{m}^{\ell}\right)>0$.

\section{SOME PRELIMINARIES}

In this paper $(R, \mathfrak{m}, k)$ will always be a Gorenstein complete local ring. We recall some basic facts on the delta invariants from [1] and [2].

For a finitely generated $R$-module $M$, an exact sequence

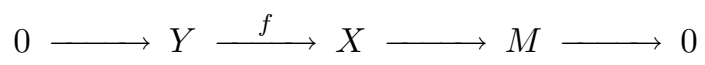

is called a Cohen-Macaulay approximation of $M$ if $X$ is a maximal Cohen-Macaulay module and $Y$ has finite projective dimension. We say that the sequence is minimal if there are no isomorphisms split out of $f$. It is known that a minimal CohenMacaulay approximation of $M$ exists uniquely up to isomorphisms.

An $R$-module is said to be stable if there is no nontrivial free direct summand. Since $R$ is a complete local ring, every finitely generated $R$-module $X$ is uniquely decomposed as a direct sum of a stable module and a free module. We denote the maximal rank of free direct summand of $X$ by $f-\operatorname{rank}_{R} X$. If the sequence (1.1) is the minimal Cohen-Macaulay approximation of $M$, then the delta invariant $\delta_{R}(M)$ is defined as f-rank $R$. It is known that $\delta_{R}(M)=0$ if and only if $M$ is a homomorphic image of a stable maximal Cohen-Macaulay module.

For an integer $n$, the $n$th delta invariant $\delta_{R}^{n}(M)$ is, by definition, the delta invariant of the $n$th syzygy module $\Omega_{R}^{n}(M)$ of $M$. Note that if $n>\operatorname{dim} R$, then $\delta_{R}^{n}(M)=0$ for any finitely generated module $M$, since $\Omega_{R}^{n}(M)$ is a stable maximal Cohen-Macaulay module.

Auslander has shown the following

Lemma (1.2) ([2, Proposition 5.7]). If $R$ is non-regular, then $\delta_{R}^{n}(k)=0$ for any $n \geqq 0$.

As a result of this lemma we have

Lemma (1.3). Suppose that $R$ is non-regular and let $M$ be a finitely generated $R$-module. Then $\delta_{R}(\mathfrak{m} M)=0$. In particular, we see that $\delta_{R}^{1}\left(R / \mathfrak{m}^{\ell}\right)=0$ for any $\ell$.

Proof. Since $\delta_{R}(\mathfrak{m})=\delta_{R}^{1}(R / \mathfrak{m})=0$, there is an epimorphism $X \longrightarrow \mathfrak{m}$ with $X$ a stable maximal Cohen-Macaulay module. If $F \longrightarrow M$ is a free cover of $M$, then we have an epimorphism $F \otimes_{R} X \longrightarrow \mathfrak{m} M$, where $F \otimes_{R} X$ is also a stable maximal Cohen-Macaulay module. Thus $\delta_{R}(\mathfrak{m} M)=0$.

Corollary (1.4). Conjecture (0.1) is true if $\operatorname{dim} R \leqq 1$.

We now remark on delta invariants under some ring extension.

Lemma (1.5). Let $\varphi: R \longrightarrow R^{\prime}$ be a local homomorphism of Gorenstein complete local rings and let $M$ be a finitely generated $R$-module. Assume that $\varphi$ is a faithfully flat morphism and $\operatorname{dim} R=\operatorname{dim} R^{\prime}$. Let the minimal Cohen-Macaulay approximation of $M$ over $R$ be given as (1.1). Then the sequence

$$
0 \longrightarrow Y \otimes_{R} R^{\prime} \stackrel{f \otimes R^{\prime}}{\longrightarrow} X \otimes_{R} R^{\prime} \longrightarrow M \otimes_{R} R^{\prime} \longrightarrow 0
$$

is the minimal Cohen-Macaulay approximation over $R^{\prime}$. 
Proof. Since $R^{\prime}$ is $R$-flat and has the same dimension as $R$, we can show that if $X$ is a maximal Cohen-Macaulay module over $R$ (resp. has finite projective dimension over $R$ ), then so is $X \otimes_{R} R^{\prime}$ over $R^{\prime}$. Thus the sequence (1.6) is a CohenMacaulay approximation of $M \otimes_{R} R^{\prime}$ over $R^{\prime}$. We have to show that (1.6) is minimal. Suppose not. Then we would have an $R^{\prime}$-homomorphism $g: X \otimes_{R} R^{\prime} \longrightarrow R^{\prime}$ such that the composition $g \cdot\left(f \otimes R^{\prime}\right)$ is an epimorphism. Since $\operatorname{Hom}_{R^{\prime}}\left(X \otimes_{R} R^{\prime}, R^{\prime}\right) \cong$ $\operatorname{Hom}_{R}(X, R) \otimes_{R} R^{\prime}$, we can write $g$ as a finite sum of $h_{i} \otimes R^{\prime}$ with $h_{i} \in \operatorname{Hom}_{R}(X, R)$. Since $R$ and $R^{\prime}$ are local rings, there is an $i$ such that $\left(h_{i} \cdot f\right) \otimes R^{\prime}$, hence $h_{i} \cdot f$, is an epimorphism. This contradicts the sequence (1.1) being minimal.

Lemma (1.7). Under the same notation as in (1.5), we have the equality

$$
\delta_{R}^{n}(M)=\delta_{R^{\prime}}^{n}\left(M \otimes_{R} R^{\prime}\right)
$$

for any integer $n \geqq 0$.

Proof. Since $R^{\prime}$ is $R$-flat, we can see that $\Omega_{R^{\prime}}^{n}\left(M \otimes_{R} R^{\prime}\right)=\Omega_{R}^{n}(M) \otimes_{R} R^{\prime}$. Thus applying Lemma (1.5), we have only to show that $f-\operatorname{rank}_{R}(X)=\mathrm{f}-\operatorname{rank}_{R^{\prime}}\left(X \otimes_{R} R^{\prime}\right)$ if $X$ is a maximal Cohen-Macaulay module over $R$. For this, it is enough to prove that if $X$ is a stable $R$-module, then $X \otimes_{R} R^{\prime}$ is also stable as an $R^{\prime}$-module. Suppose $X \otimes_{R} R^{\prime}$ is not stable over $R^{\prime}$. Then we have an epimorphism $g: X \otimes_{R} R^{\prime} \longrightarrow R^{\prime}$. As in the proof of (1.5) we can write $g$ as a finite sum of $h_{i} \otimes R^{\prime}$ with $h_{i} \in \operatorname{Hom}_{R}(X, R)$. Then $h_{i}$ is an epimorphism for some $i$. This contradicts that $X$ is stable.

The same equality as in (1.7) is discussed in a recent work of Shida [7]. The following lemma is necessary for the proof of our theorem:

Lemma (1.8). Let $x \in \mathfrak{m}$ be a non zero-divisor both on $R$ and on a finitely generated $R$-module $M$. Putting $\bar{R}=R / x R$, we have the equality $\delta_{\bar{R}}\left(M \otimes_{R} \bar{R}\right)=\delta_{R}(M)$.

This lemma follows easily from [2, Lemma 5.1].

\section{MAIN THEOREMS}

In the rest of the paper, $(R, \mathfrak{m}, k)$ is a complete Gorenstein local ring of dimension $d$, and we always assume that $R$ is non-regular. Our main theorems in this paper are the following:

Theorem (2.1). There is an integer $\ell_{0}$ such that $\delta_{R}^{n}\left(R / \mathfrak{m}^{\ell}\right)=0$ for any $\ell \geqq \ell_{0}$ and for any $n>0$.

Theorem (2.2). Let $G=g r_{\mathfrak{m}}(R)$ be the associated graded ring of $R$ with respect to the maximal ideal. If $\operatorname{depth} G=t$, then we have $\delta_{R}^{n}\left(R / \mathfrak{m}^{\ell}\right)=0$ for any $\ell>0$ and for any $n \geqq d-t+1$.

As a consequence of the above theorems, we see that there are only finitely many possibilities for a pair of positive integers $(n, \ell)$ with $\delta_{R}^{n}\left(R / \mathfrak{m}^{\ell}\right)>0$. Furthermore we have the following corollary as a direct consequence of $(2.2)$, which generalizes a result of Herzog [6, Cor. (2.4)].

Corollary (2.3). Suppose that the associated graded ring $G$ has depth $\geqq d-1$. (For example, $R$ is either a ring of hypersurface or a homogeneous graded ring.) Then $\delta_{R}^{\ell}\left(R / \mathfrak{m}^{n}\right)=0$ for any positive integers $n$ and $\ell$.

Proof. Apply (2.2) to get $\delta_{R}^{n}\left(R / \mathfrak{m}^{\ell}\right)=0$ for $n \geqq 2$, then combine it with (1.3).

Before proceeding to the proof, we make the following 
Remark 2.4. When proving Theorems (2.1) and (2.2), we may assume that the residue field $k$ is an infinite field.

To show this, let $u$ be an indeterminate and let $R^{\prime}$ be the completion of the local $\operatorname{ring} R[u]_{\mathfrak{m}[u]}$. Then $R^{\prime}$ is a faithfully flat $R$-algebra, which is a complete Gorenstein local ring with maximal ideal $\mathfrak{m}^{\prime}=\mathfrak{m} R^{\prime}$ and with residue field $k^{\prime}=k(u)$ that is, in fact, an infinite field. Notice in this setting that $R^{\prime} / \mathfrak{m}^{\prime \ell}=R / \mathfrak{m}^{\ell} \otimes_{R} R^{\prime}$. Hence it follows from (1.7) that $\delta_{R^{\prime}}^{n}\left(R^{\prime} / \mathfrak{m}^{\prime \ell}\right)=\delta_{R}^{n}\left(R / \mathfrak{m}^{\ell}\right)$. Note also that $g r_{\mathfrak{m}^{\prime}}\left(R^{\prime}\right)=$ $g r_{\mathfrak{m}}(R) \otimes_{R} R^{\prime}$, in particular, one can show depth $g r_{\mathfrak{m}^{\prime}}\left(R^{\prime}\right)=\operatorname{depth} g r_{\mathfrak{m}}(R)$. Thus, if necessary, taking $R^{\prime}$ instead of $R$, we may assume that $R$ has an infinite residue field.

We need a lemma to prove the theorems.

Lemma (2.5). Let $M$ be a finitely generated $R$-module and let $x \in \mathfrak{m}$ be a nonzero-divisor on $R$. Suppose that the initial form $x^{*}$ of $x$ in $G=g r_{\mathfrak{m}}(R)$ is a non-zero divisor on $g r_{\mathfrak{m}}(M)$. Furthermore we denote $\bar{R}=R / x R$ and $\bar{M}=M / x M$. Then we have the following isomorphism for each $n \geqq 0$ :

$$
\Omega_{R}^{n}(\mathfrak{m} M) \otimes_{R} \bar{R} \cong \Omega \frac{n}{\bar{R}}(\mathfrak{m} \bar{M}) \oplus \Omega \frac{n}{R}(M / \mathfrak{m} M) .
$$

Proof. We note from the assumption that $x$ is a non-zero divisor on $M$ and the multiplication map $M / \mathfrak{m} M \rightarrow \mathfrak{m} M / \mathfrak{m}^{2} M$ of $x$ is an injective map. Thus we have the following commutative diagram with an exact row:

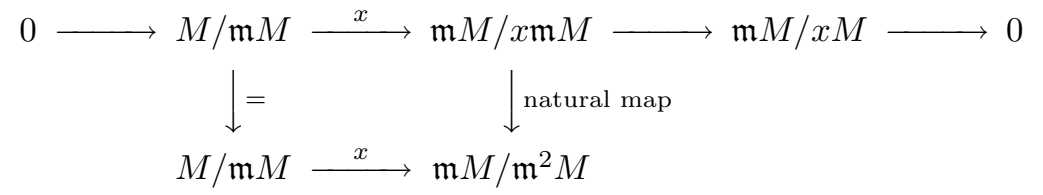

As the map in the second row is an injective map of $k$-vector spaces, it is split and thus the first row is a split exact sequence. Therefore we see that

$$
\mathfrak{m} M \otimes_{R} \bar{R} \cong \mathfrak{m}(M / x M) \oplus M / \mathfrak{m} M .
$$

Now we prove the lemma by induction on $n$. For $n=0$ the lemma is nothing but the above isomorphism. For $n>0$, we take the minimal free cover of $\Omega_{R}^{n-1}(\mathfrak{m} M)$ to get the exact sequence:

$$
0 \longrightarrow \Omega_{R}^{n}(\mathfrak{m} M) \longrightarrow F \longrightarrow \Omega_{R}^{n-1}(\mathfrak{m} M) \longrightarrow 0 .
$$

Taking the tensor product of this sequence with $\bar{R}$, we can show that the sequence

$$
0 \longrightarrow \Omega_{R}^{n}(\mathfrak{m} M) \otimes_{R} \bar{R} \longrightarrow F \otimes_{R} \bar{R} \longrightarrow \Omega_{R}^{n-1}(\mathfrak{m} M) \otimes_{R} \bar{R} \longrightarrow 0
$$

gives a minimal free cover over $\bar{R}$. Thus we have an isomorphism

$$
\Omega_{R}^{n}(\mathfrak{m} M) \otimes_{R} \bar{R} \cong \Omega \frac{1}{R}\left(\Omega_{R}^{n-1}(\mathfrak{m} M) \otimes_{R} \bar{R}\right) .
$$

The lemma follows from this isomorphism together with the induction hypothesis.

We should mention that a reduction argument similar to that used in this lemma already appeared in Ding [5].

Proof of Theorem (2.1). In this proof we assume that $k$ is an infinite field by (2.4). We prove the theorem by induction on $d=\operatorname{dim} R$. If $d \leqq 1$, then the theorem holds by (1.4). 
Now suppose $d>1$ and we denote the associated graded ring of $R$ with respect to the maximal ideal by $G=g r_{\mathfrak{m}}(R)$ and the irrelevant maximal ideal of $G$ by $G_{+}$. Let $I=\mathrm{H}_{G_{+}}^{0}(G)$ be the ideal of $G$ consisting of all elements which are annihilated by some powers of $G_{+}$. Note that $I$ is a graded ideal of finite length, hence there is an integer $\ell_{1}$ such that $I_{\ell}=(0)$ for any $\ell \geqq \ell_{1}$. Since $G / I$ has positive depth and since $k$ is an infinite field, we can find an element $x \in \mathfrak{m}-\mathfrak{m}^{2}$ so that the initial form $x^{*} \in G$ is a non-zero divisor on $G / I$. Then, for $\ell \geqq \ell_{1}$, the multiplication of $x$ induces the injective map

$$
\mathfrak{m}^{\ell} / \mathfrak{m}^{\ell+1} \longrightarrow \mathfrak{m}^{\ell+1} / \mathfrak{m}^{\ell+2},
$$

since $(G / I)_{\ell}=G_{\ell}$. In particular, one sees that $x^{*}$ is a non-zero-divisor on the $G$ module $g r_{\mathfrak{m}}\left(\mathfrak{m}^{\ell}\right)$ for $\ell \geqq \ell_{1}$. Thus we can apply Lemma (2.5) to get the isomorphism:

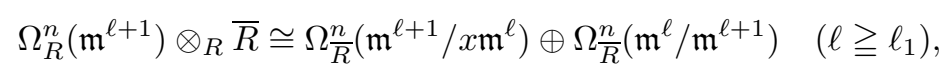

where $\bar{R}=R / x R$.

Let $\overline{\mathfrak{m}}$ be the maximal ideal $\mathfrak{m} / x R$ of $\bar{R}$. We shall show that there is an integer $\ell_{2}\left(\geqq \ell_{1}\right)$ such that $\overline{\mathfrak{m}}^{\ell+1}=\mathfrak{m}^{\ell+1} / x \mathfrak{m}^{\ell}$ for any $\ell \geqq \ell_{2}$. To show this, it is enough to prove $x R \cap \mathfrak{m}^{\ell+1}=x \mathfrak{m}^{\ell}$ for $\ell \geqq \ell_{2}$, since $\overline{\mathfrak{m}}^{\ell+1}=\mathfrak{m}^{\ell+1} / x R \cap \mathfrak{m}^{\ell+1}$. By the lemma of Artin-Rees, we know that there is an integer $r(>0)$ such that $x R \cap \mathfrak{m}^{\ell+1}=$ $\mathfrak{m}^{\ell+1-r}\left(x R \cap \mathfrak{m}^{r}\right)$ for $\ell \geqq r$. Hence

$$
x R \cap \mathfrak{m}^{\ell+1}=x \mathfrak{m}^{\ell+1-r} \cap \mathfrak{m}^{\ell+1},
$$

for such $\ell$. Now take $\ell$ such that $\ell \geqq \ell_{1}+r-1$. Then, since $\ell+i-r \geqq \ell_{1}$ for $i \geqq 1$, we see that $(G / I)_{\ell+i-r}=G_{\ell+i-r}$ for $i \geqq 1$. Thus the multiplication by $x$ induces the following sequence of injective maps:

$$
\mathfrak{m}^{\ell+1-r} / \mathfrak{m}^{\ell+2-r} \longrightarrow \mathfrak{m}^{\ell+2-r} / \mathfrak{m}^{\ell+3-r} \longrightarrow \cdots \longrightarrow \mathfrak{m}^{\ell-1} / \mathfrak{m}^{\ell} \longrightarrow \mathfrak{m}^{\ell} / \mathfrak{m}^{\ell+1} .
$$

This means that the right hand side of (2.7) equals $x \mathrm{~m}^{\ell}$. Therefore we have shown the equality $\overline{\mathfrak{m}}^{\ell+1}=\mathfrak{m}^{\ell+1} / x \mathfrak{m}^{\ell}$ holds for $\ell \geqq \ell_{2}:=\ell_{1}+r-1$. Combining this with (2.6), we thus have

$$
\Omega_{R}^{n}\left(\mathfrak{m}^{\ell+1}\right) \otimes_{R} \bar{R} \cong \Omega_{\frac{n}{R}}\left(\overline{\mathfrak{m}}^{\ell+1}\right) \oplus \Omega \frac{n}{R}\left(\mathfrak{m}^{\ell} / \mathfrak{m}^{\ell+1}\right),
$$

for $\ell \geqq \ell_{2}$.

Now by the induction hypothesis, we find an integer $\ell_{3}$ such that

$$
\delta_{\bar{R}}\left(\Omega \frac{n}{R}\left(\overline{\mathfrak{m}}^{\ell+1}\right)\right)=\delta \bar{R}^{n}\left(\overline{\mathfrak{m}}^{\ell+1}\right)=0,
$$

for $\ell \geqq \ell_{3}$. On the other hand, we know from (1.2) that $\delta \frac{n}{R}\left(\mathfrak{m}^{\ell} / \mathfrak{m}^{\ell+1}\right)=0$ for any $\ell$ and $n$. Thus it follows from $(2.8)$ that $\delta_{\bar{R}}\left(\Omega_{R}^{n}\left(\mathfrak{m}^{\ell+1}\right) \otimes_{R} \bar{R}\right)=0$ for $\ell \geqq \ell_{0}:=$ $\max \left\{\ell_{2}, \ell_{3}\right\}$. Then it follows from (1.8) that $\delta_{R}^{n}\left(\mathfrak{m}^{\ell+1}\right)=\delta_{R}\left(\Omega_{R}^{n}\left(\mathfrak{m}^{\ell+1}\right)\right)=0$ for $\ell \geqq \ell_{0}$ as desired.

Proof of Theorem (2.2). The proof of this theorem goes through in the same way as the proof of (2.1). We may assume that $k$ is an infinite field. We prove the theorem by induction on $t=\operatorname{depth} G$. If $t=0$, then the theorem obviously holds since $\delta_{R}^{n}(M)=0$ for any $n>d=\operatorname{dim} R$ and for any finitely generated $R$-module $M$. Assume $t \geqq 1$. Since $k$ is infinite, there is an element $x \in \mathfrak{m}-\mathfrak{m}^{2}$ such that the initial form $x^{*} \in G$ is a non-zero-divisor on $G$. For a fixed integer $\ell$, we can apply 
Lemma (2.5) to $M=\mathfrak{m}^{\ell-1}$, since $x^{*}$ is also a non-zero-divisor on $g r_{\mathfrak{m}}\left(\mathfrak{m}^{\ell-1}\right) \subseteq G$. Thus we have an isomorphism for each $n>0$ :

$$
\Omega_{R}^{n-1}\left(\mathfrak{m}^{\ell}\right) \otimes_{R} \bar{R} \cong \Omega_{\bar{R}}^{n-1}\left(\overline{\mathfrak{m}}^{\ell}\right) \oplus \Omega_{\bar{R}}^{n-1}\left(\mathfrak{m}^{\ell-1} / \mathfrak{m}^{\ell}\right),
$$

where $\bar{R}=R / x R$ and $\overline{\mathfrak{m}}=\mathfrak{m} / x R$. Note in this case that we have $\mathfrak{m}^{\ell+1} / x \mathfrak{m}^{\ell}=\overline{\mathfrak{m}}^{\ell+1}$ for any $\ell \geqq 0$, since $x^{*}$ is a non-zero divisor on $G$. Compare with the proof of (2.1). We also notice that $g r_{\overline{\mathfrak{m}}}(\bar{R})=G / x^{*} G$, in particular, depth $g r_{\overline{\mathfrak{m}}}(\bar{R})=t-1$. Thus by the induction hypothesis, we see that

$$
\delta_{\bar{R}}\left(\Omega_{\bar{R}}^{n-1}\left(\overline{\mathfrak{m}}^{\ell}\right)\right)=\delta_{\bar{R}}\left(\Omega_{\bar{R}}^{n}\left(\bar{R} / \overline{\mathfrak{m}}^{\ell}\right)\right)=\delta_{\bar{R}}^{n}\left(\bar{R} / \overline{\mathfrak{m}}^{\ell}\right)=0,
$$

for any $n \geqq(d-1)-(t-1)+1=d-t+1$. On the other hand, we know from (1.2) that $\delta_{\bar{R}}\left(\Omega_{\bar{R}}^{n-1}\left(\mathfrak{m}^{\ell-1} / \mathfrak{m}^{\ell}\right)\right)=\delta_{\bar{R}}^{n-1}\left(\mathfrak{m}^{\ell-1} / \mathfrak{m}^{\ell}\right)=0$ for any $n>0$. Therefore it follows from (2.9) that $\delta_{\bar{R}}\left(\Omega_{R}^{n-1}\left(\mathfrak{m}^{\ell}\right) \otimes_{R} \bar{R}\right)=0$ if $n \geqq d-t+1$. Thus Lemma (1.8) implies that $\delta_{R}^{n}\left(R / \mathfrak{m}^{\ell}\right)=\delta_{R}^{n-1}\left(\mathfrak{m}^{\ell}\right)=\delta_{R}\left(\Omega_{R}^{n-1}\left(\mathfrak{m}^{\ell}\right)\right)=0$ as desired.

\section{Some REMARKS FOR TWO-DIMENSIONAL CASES}

In this section we assume that the Gorenstein complete local ring $(R, \mathfrak{m}, k)$ has dimension 2 . We denote the associated graded ring $g r_{\mathfrak{m}}(R)$ by $G$, as before.

As we have shown in (2.2), if $G$ has positive depth, then the conjecture (0.1) is true for $R$. However there is, of course, a Gorenstein local ring $R$ with $\operatorname{depth} G=0$. One of the easiest examples is

$$
R=k[[u, v, w, x, y]] /\left(u^{2}, u x-v^{3}, u y-w^{3}\right),
$$

for which I do not know if the conjecture is true or not.

If one wants to make a counterexample to the conjecture (0.1), the following lemma will be useful.

Lemma (3.1). The following two conditions are equivalent for an $\mathfrak{m}$-primary ideal I.

(a) $\delta_{R}^{2}(R / I)>0$.

(b) There is an exact sequence

$$
0 \longrightarrow R \stackrel{j}{\longrightarrow} L \stackrel{p}{\longrightarrow} I \longrightarrow 0
$$

with $p \otimes_{R} k$ an isomorphism (or equivalently $j(1) \in \mathfrak{m} L$ ).

Proof. $(a) \Longrightarrow(b)$ Since $M:=\Omega^{2}(R / I)$ is a maximal Cohen-Macaulay module over $R$, the condition $(a)$ says exactly that $M$ contains a free module as a direct summand. Thus there is an epimorphism $\rho: M \longrightarrow R$ and we have a push-out diagram

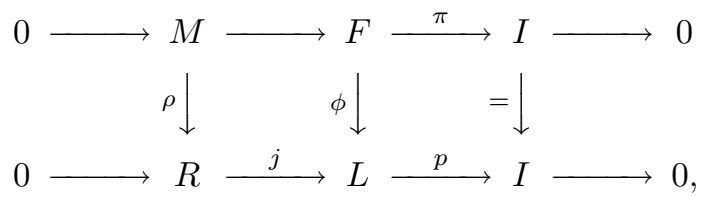

where $\pi$ is a minimal free cover of $I$. Since $\pi=p \cdot \phi$, we see that $(p \otimes k) \cdot(\phi \otimes k)=\pi \otimes k$ is an isomorphism. Note from the diagram that $\phi \otimes k$ is an epimorphism and we have that $p \otimes k$ is an isomorphism. 
$(b) \Longrightarrow(a)$ Let $\phi: F \longrightarrow L$ be a minimal free cover of $L$. Then we have the commutative diagram:

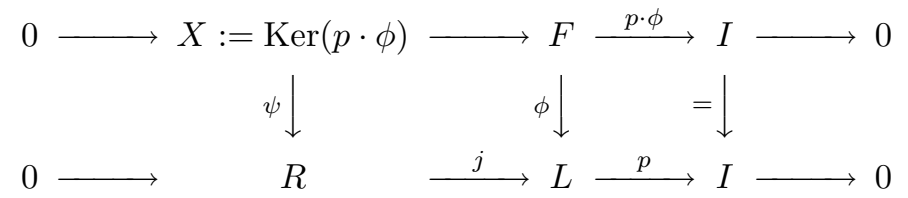

Since $(p \cdot \phi) \otimes k=(p \otimes k) \cdot(\phi \otimes k)$ is an isomorphism, we can see that $p \cdot \phi$ is a minimal free cover of $I$. Thus we have $X \cong \Omega_{R}^{2}(R / I)$. On the other hand, it follows from the diagram that $\psi$ is an epimorphism and that $\Omega_{R}^{2}(R / I)$ has $R$ as a direct summand.

As one of the consequences of (3.1), we get the following

Proposition (3.2). Suppose that there exist an integer $r$ and a system of parameters $\{x, y\}$ of $R$ which satisfy the following conditions:

(a) $(x, y) \subseteq \mathfrak{m}^{r+1}$.

(b) $(x, y) \mathfrak{m}^{r}=\mathfrak{m}^{2 r+1}$.

Then we have $\delta_{R}^{2}\left(R / \mathfrak{m}^{2 r+1}\right)>0$.

Proof. Let $I=(x, y) R$ and take the free resolution of $I$ :

$$
0 \longrightarrow R \stackrel{f_{2}}{\longrightarrow} R^{2} \stackrel{f_{1}}{\longrightarrow} I \longrightarrow 0,
$$

which is part of the Koszul complex. Then we see that $\operatorname{Im}\left(f_{2}\right) \subseteq I R^{2} \subseteq \mathfrak{m}^{r+1} R^{2}$. Thus we have the exact sequence

$$
0 \longrightarrow R \stackrel{f_{2}}{\longrightarrow} \mathfrak{m}^{r} R^{2} \stackrel{f_{1}^{\prime}}{\longrightarrow} \mathfrak{m}^{r} I \longrightarrow 0,
$$

where $f_{1}^{\prime}$ is the restriction to $f_{1}$ on $\mathfrak{m}^{r} R^{2}$. Since $f_{2}(1) \in \mathfrak{m}\left(\mathfrak{m}^{r} R^{2}\right)$, it follows from (3.1) that $\delta_{R}^{2}\left(R / \mathfrak{m}^{r} I\right)>0$.

If $R$ is a regular local ring, then the regular system of parameters satisfies the conditions in (3.2) for $r=0$. For a non-regular ring $R$, if there is a system of parameters satisfying the conditions $(a),(b)$ in (3.4), then we must have $\operatorname{depth} G=0$ by (2.2). We do not know if there is a non-regular Gorenstein local ring with the conditions in (3.4), or not.

\section{REFERENCES}

[1] M. Auslander, R. -O. Buchweitz, The homological theory of maximal Cohen-Macaulay approximations, Soc. Math. de France, Mem. 38 (1989), 5-37. MR 91h:13010

[2] M. Auslander, S. Ding, Ø. Solberg, Liftings and weak liftings of modules, J. Algebra 156 (1993), 273-317. MR 94d:16007

[3] S. Ding, Cohen-Macaulay approximations and multiplicities, J. Algebra 153 (1992), 172-188. MR 94c: 13024

[4] S. Ding, A note on the index of Cohen-Macaulay local rings, Comm. Algebra 21 (1993), 53-71. MR 94b:13014

[5] S. Ding, The associated graded ring and the index of a Gorenstein local ring, Proc. of the Amer. Math. Soc. 120 (1994), 1029-1033. MR 94f:13014

[6] J. Herzog, On the index of a homogenous Gorenstein ring, Contemporary Math. 159 (1994), 95-102. MR 95b:13031

[7] A. Shida, On indices of local rings along local homomorphisms, Preprint, Nagoya Univ. (1994).

Institute of Mathematics, Faculty of Integrated Human Studies, Kyoto University, Yoshida-Nihonmatsu, SAKYo-Ku, KyOto 606-01, JAPAN

E-mail address: yoshino@math.h.kyoto-u.ac.jp 